\title{
Delivering the world's most intense muon beam
}

\author{
S. Cook,${ }^{1}$ R. D’Arcy, ${ }^{1}$ A. Edmonds, ${ }^{1}$ M. Fukuda, ${ }^{2}$ K. Hatanaka, ${ }^{2}$ Y. Hino, ${ }^{3}$ Y. Kuno, ${ }^{3}$ \\ M. Lancaster, ${ }^{1}$ Y. Mori, ${ }^{4}$ T. Ogitsu, ${ }^{5}$ H. Sakamoto, ${ }^{3}$ A. Sato, ${ }^{3}$ N. H. Tran, ${ }^{3}$ N. M. Truong, ${ }^{3}$ \\ M. Wing, ${ }^{1, *}$ A. Yamamoto, ${ }^{5}$ and M. Yoshida ${ }^{5}$ \\ ${ }^{1}$ Department of Physics and Astronomy, UCL, Gower Street, London WC1E 6BT, United Kingdom \\ ${ }^{2}$ Research Center for Nuclear Physics (RCNP), Osaka University, Osaka 567-0047, Japan \\ ${ }^{3}$ Department of Physics, Graduate School of Science, Osaka University, Osaka 569-0043, Japan \\ ${ }^{4}$ Kyoto University Reactor Research Institute (KURRI), Kyoto 590-0494, Japan \\ ${ }^{5}$ High Energy Accelerator Research Organization (KEK), Tsukuba 305-0801, Japan
}

(Received 25 October 2016; published 15 March 2017)

\begin{abstract}
A new muon beam line, the muon science innovative channel, was set up at the Research Center for Nuclear Physics, Osaka University, in Osaka, Japan, using the $392 \mathrm{MeV}$ proton beam impinging on a target. The production of an intense muon beam relies on the efficient capture of pions, which subsequently decay to muons, using a novel superconducting solenoid magnet system. After the pion-capture solenoid, the first $36^{\circ}$ of the curved muon transport line was commissioned and the muon flux was measured. In order to detect muons, a target of either copper or magnesium was placed to stop muons at the end of the muon beam line. Two stations of plastic scintillators located upstream and downstream from the muon target were used to reconstruct the decay spectrum of muons. In a complementary method to detect negatively charged muons, the $\mathrm{x}$-ray spectrum yielded by muonic atoms in the target was measured in a germanium detector. Measurements, at a proton beam current of $6 \mathrm{pA}$, yielded $(10.4 \pm 2.7) \times 10^{5}$ muons per watt of proton beam power $\left(\mu^{+}\right.$and $\left.\mu^{-}\right)$, far in excess of other facilities. At full beam power $(400 \mathrm{~W})$, this implies a rate of muons of $(4.2 \pm 1.1) \times 10^{8}$ muons s${ }^{-1}$, among the highest in the world. The number of $\mu^{-}$measured was about a factor of 10 lower, again by far the most efficient muon beam produced. The setup is a prototype for future experiments requiring a high-intensity muon beam, such as a muon collider or neutrino factory, or the search for rare muon decays which would be a signature for phenomena beyond the Standard Model of particle physics. Such a muon beam can also be used in other branches of physics, nuclear and condensed matter, as well as other areas of scientific research.
\end{abstract}

DOI: 10.1103/PhysRevAccelBeams.20.030101

\section{INTRODUCTION}

High-intensity muon beams have applications in many areas of science, spanning high-energy particle physics to condensed matter physics and even areas of chemistry and biology. Many results are limited by statistics, and, depending on the experiment, up to and above $10^{18}$ muons per year are required, whereas only $10^{15}$ muons per year are available now.

In particle physics, intense muon beams are needed for the following experiments and areas of investigation. Rare muon decays such as charged lepton flavor violation (CLFV) have attracted much attention theoretically and experimentally $[1,2]$. As the Standard Model (SM) expectation for such processes is so small $\left[\sim O\left(10^{-54}\right)\right]$, higherintensity muon beams could lead to the unequivocal

\footnotetext{
*Corresponding author. m.wing@ucl.ac.uk

Published by the American Physical Society under the terms of the Creative Commons Attribution 4.0 International license. Further distribution of this work must maintain attribution to the author(s) and the published article's title, journal citation, and DOI.
}

discovery of physics beyond the SM. There are several current and planned experiments searching for CLFV with muons. They are, for example, $\mu \rightarrow e \gamma$ [3], $\mu-e$ conversion in a muonic atom [4,5], and $\mu \rightarrow$ eee [6]. In particular, planned experiments of COMET [4] in Japan and Mu2e [5] in the United States, which will search for $\mu-e$ conversion with anticipated improvement of physics sensitivity of $10^{4}$, need high-intensity muon beams of $10^{18}$ muons per year. The properties of the muon such as its mean lifetime, which gives a direct determination of the Fermi constant, or anomalous magnetic moment have both been measured to a precision of about one part per million [7-9]. Given the approximate $3 \sigma$ difference between the theory and data in the measurement [8] of the anomalous magnetic moment, new experiments to measure with a factor of 4 better precision are currently under construction $[10,11]$. Highly intense muon beams of $10^{21}$ muons per year are needed for a muon collider, a machine that can investigate the energy frontier, i.e. the $\mathrm{TeV}$ scale. A muon collider has a number of advantages such as compactness and lower synchrotron radiation compared to an $e^{+} e^{-}$ collider but also has a number of technical challenges [12]. 
The use of muon beams in condensed matter physics, in particular, as probes of the magnetic properties of materials, is given in detail elsewhere $[13,14]$. Muons from the decay of charged pions at rest are naturally $100 \%$ polarized and are subsequently stopped by the material under investigation. Internal magnetic fields inside the material can be studied by precession of the muon spin, which can be detected from the time-dependent angular distribution of the emitted electrons and so does not involve scattering as neutron or x-ray material probes do. Also, x-ray emission spectra from negative muons captured in matter provide a nondestructive method of determining the elemental content of a given sample, e.g., the characterization of an archaeological artifact $[15,16]$.

Muon beams are usually produced via the decay of a large number of charged pions, produced by colliding a proton with a fixed target. The challenges of producing high-intensity muon beams are the need for a high-power proton beam; the efficient capture of pions produced at the target; and, given the large number of particles produced with a wide range of kinematic properties, effective methods to achieve a pure muon beam. Often, muon facilities and neutron facilities are combined. The pion production target for a muon beam requires a small beam loss as a neutron production target is located downstream; in such cases, a short target and no magnetic field surrounding the target are employed. At the muon science innovative channel (MuSIC), which is a dedicated muon source, a relatively long target is used, and coupled with a system of superconducting solenoid magnets [17], a high muon production efficiency is achieved. The scheme to capture pions using solenoid magnets was first discussed by the MELC experiment $[18,19]$ and is also proposed for muon conversion experiments [4,5], muon colliders [12], and neutrino factories [20]. Additionally, unlike particles usually used in particle accelerators, muons have a finite lifetime of $2.2 \mu \mathrm{s}$, and so methods are needed to store them before decay. The most intense muon beam produced is the $\mu \mathrm{E} 4$ beam line at the Paul Scherrer Institut in Switzerland, which is capable of producing $J_{\mu}=4 \times 10^{8}$ muons s $^{-1}$ with the momenta of about $28 \mathrm{MeV}$ [21]. Given an initial proton beam power of $W_{p}=1.2 \mathrm{MW}$, this equates to $J_{\mu} / W_{p}=3.5 \times 10^{2}$ muons s ${ }^{-1} \mathrm{~W}^{-1}$.

In this article, a new high-intensity muon beam line, MuSIC, at the Research Center for Nuclear Physics (RCNP), Osaka University, in Osaka, Japan, is presented. The article reports on the commissioning of the beam line and a measure of the intensity of muons produced; the facility can be used for much of the variety of science discussed above.

\section{HIGH-INTENSITY MUON SOURCE}

High-intensity muon sources require the collection of many pions which will produce muons in their decays. To collect as many pions (and cloud muons) as possible, the pions are captured using a high-strength solenoidal magnetic field giving a large solid angle acceptance. The pion capture system consists of the pion production target, high-field solenoid magnets for pion capture, and a radiation shield. In the MuSIC case, pions emitted into the backward hemisphere can be captured within a transverse momentum threshold $p_{\mathrm{T}}^{\max }$. This $p_{\mathrm{T}}^{\max }$ is given by the magnetic field strength $B$ and the radius of the inner bore of the solenoid magnet, $R$, as

$$
p_{\mathrm{T}}^{\max }(\mathrm{GeV} / c)=0.3 \times B(\mathrm{~T}) \times \frac{R(\mathrm{~m})}{2} .
$$

The target is located at the position of the maximum magnetic field to maximize the solid angle for pions. It is known that the higher the pion capture magnetic field, the better the muon yield at the exit of the muon beam line. In addition, the beam emittance is better if a higher magnetic field is used for the pion capture. Therefore, a higher magnetic field is preferable.

The pions captured at the pion capture system have a broad directional distribution. In order to increase the acceptance of the muon beam line, it is desirable to make them more parallel to the beam axis by changing the magnetic field adiabatically. From the angular momentum conservation, under a solenoidal magnetic field, the product of the radius of curvature $R$ and the transverse momentum $p_{\mathrm{T}}$ is an invariant:

$$
p_{\mathrm{T}} \times R \propto \frac{p_{\mathrm{T}}^{2}}{B}=\text { const }
$$

where $B$ is the magnitude of the magnetic field. Therefore, if the magnetic field decreases gradually, $p_{\mathrm{T}}$ also decreases, yielding a more parallel beam. This is the principle of the adiabatic transition. Quantitatively, when the magnetic field is reduced by a factor of 1.75 (as for the MuSIC case, where a magnetic field of $3.5 \mathrm{~T}$ is changed into $2 \mathrm{~T}$ ), $p_{\mathrm{T}}$ decreases by a factor of $\sqrt{1.75}$. On the other hand, since

$$
p_{\mathrm{T}} \times R \propto B \times R^{2}=\text { const }^{\prime},
$$

the radius of curvature increases by a factor of $\sqrt{1.75}$. Therefore, the inner radius of the magnet in the muon transport section has to be $\sqrt{1.75}$ times that of the pion capture solenoid (or, more precisely, the inner radius of the radiation shielding of the pion capture solenoid). At the cost of an increased beam size, the pion beam can be made more parallel.

The selection of an electric charge and momenta of beam particles can be performed by using curved (toroidal) solenoids, which makes the beam dispersive. A charged particle in a solenoidal field will follow a helical trajectory. 
In a curved solenoid, the central axis of this trajectory drifts in the direction perpendicular to the plane of curvature. The magnitude of this drift, $D$, is given by

$$
\begin{aligned}
D & =\frac{1}{q B}\left(\frac{s}{R}\right) \frac{p_{\mathrm{L}}^{2}+\frac{1}{2} p_{\mathrm{T}}^{2}}{p_{\mathrm{L}}} \\
& =\frac{1}{q B}\left(\frac{s}{R}\right) \frac{p}{2}\left(\cos \theta+\frac{1}{\cos \theta}\right),
\end{aligned}
$$

where $q$ is the electric charge of the particle (with its sign), $B$ is the magnetic field at the axis, and $s$ and $R$ are the path length and the radius of curvature of the curved solenoid, respectively. Here, $s / R\left(=\theta_{\text {bend }}\right)$ is the total bending angle of the solenoid; hence, $D$ is proportional to $\theta_{\text {bend }}$. The quantities $p_{\mathrm{L}}$ and $p_{\mathrm{T}}$ are longitudinal and transverse momenta, respectively, where $\theta$ is the pitch angle of the helical trajectory. Because of the dependence on $q$, charged particles with opposite signs move in opposite directions. This can be used for charge and momentum selection if a suitable collimator is placed after the curved solenoid.

To keep the center of the helical trajectories of muons with a reference momentum $p_{0}$ in the bending plane, a correction dipole $(\mathrm{CD})$ field parallel to the drift direction can be applied. If a correction dipole field $B_{\mathrm{CD}}$, given by

$$
B_{\mathrm{CD}}=\frac{1}{q R} \frac{p_{0}}{2}\left(\cos \theta_{0}+\frac{1}{\cos \theta_{0}}\right)
$$

is applied, the trajectories of particles of charge $q$ with momentum $p_{0}$ and pitch angle $\theta_{0}$ will be corrected to be on axis.

\section{MUON PRODUCTION AT RCNP}

The proton cyclotron accelerator at RCNP, Osaka University, Japan, provides a continuous proton beam [22]. Protons are produced in an ion source and accelerated in two stages, initially up to about $65 \mathrm{MeV}$ and finally up to about $400 \mathrm{MeV}$. A maximum beam current of $1 \mu \mathrm{A}$, corresponding to a beam power of $400 \mathrm{~W}$, can be transported to the MuSIC experimental facility. The results presented here are based on a beam energy of $392 \mathrm{MeV}$ and a proton beam current $I_{p}$ in the range from $6 \mathrm{pA}$ to $1 \mathrm{nA}$ measured during data taking using monitors at the end of the beam line.

The proton beam impacts a graphite cylindrical target of length $20 \mathrm{~cm}$ and radius $2 \mathrm{~cm}$, at an angle $22^{\circ}$ horizontally from the surrounding pion capture solenoid (PCS) axis (see Fig. 1) so that the proton beam trajectory and the target axis are aligned. Fluorescent plates are attached to both ends of the target circular surface so that, by looking at the fluorescent light, the beam can be centered on the target face. The target is supported by a support shaft of $5 \mathrm{~m}$ in length and can be removed or inserted easily. The target is surrounded by stainless steel shielding of up to $27 \mathrm{~cm}$ thick, tapering on either side of the target. The taper is more rapid in the backwards direction (see Fig. 1) in order to capture the maximum number of pions and muons.

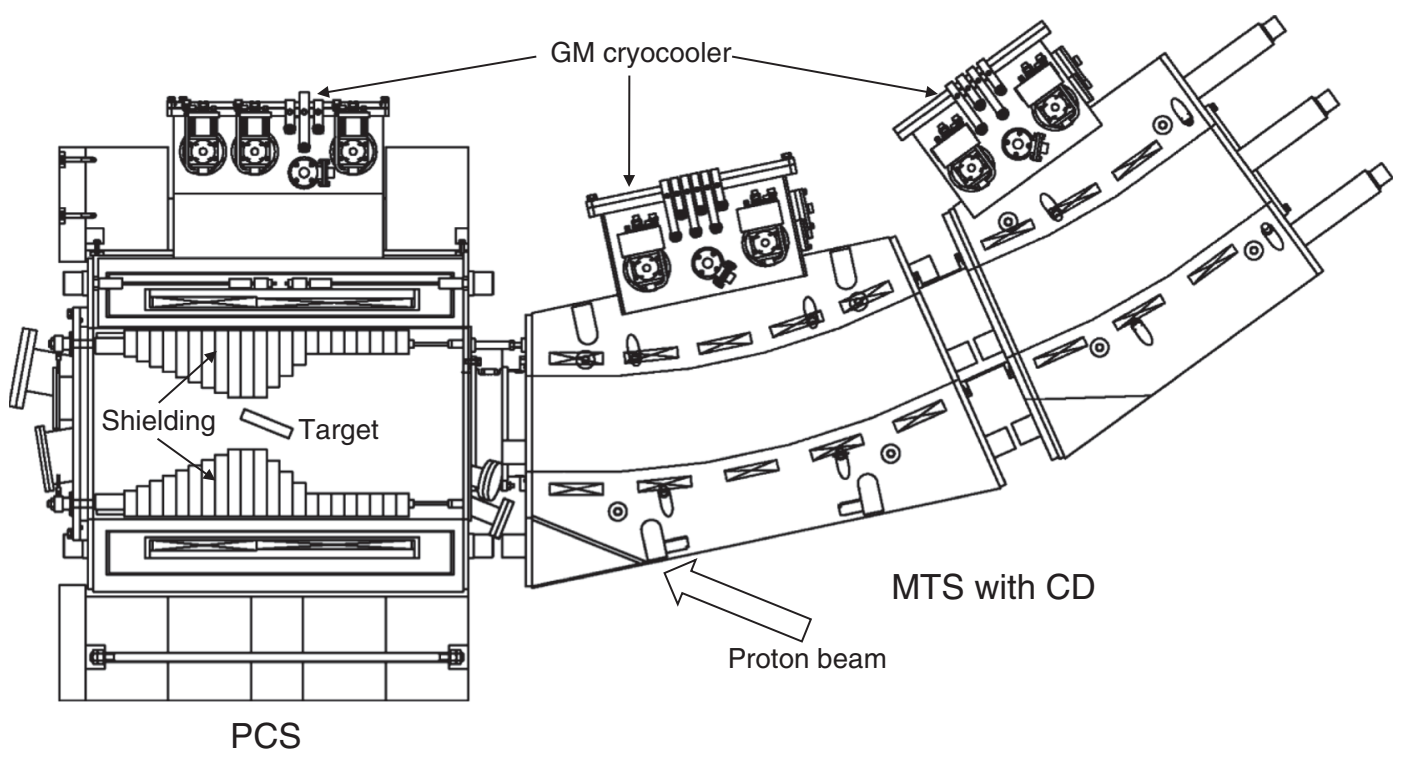

FIG. 1. Layout of the MuSIC beam line showing the target surrounded by shielding and the PCS. To the right are the two modules of five and three element coils, respectively, of the MTS, covering an arc of $36^{\circ}$. Each coil in the MTS includes a correction dipole (CD). All superconducting coils are cooled with conduction cooling using Gifford-McMahon (GM) cryocoolers. The direction of the incoming proton beam is also indicated. 
To reduce backgrounds from, in particular, neutrons and protons, which will primarily be emitted in the forward (direction of the initial proton beam) direction, backwardgoing pions are collected. Backward-going pions are captured by the PCS with a peak magnetic field of $3.5 \mathrm{~T}$ and focused towards the muon transport solenoid (MTS) via the graded magnetic field. The design parameters of the PCS are given in Table I [17].

The copper-stabilized NbTi superconducting coils are cooled using three GM cryocoolers which have a total cooling power of $4 \mathrm{~W}$ at the operating temperature of $4 \mathrm{~K}$. Nuclear heating, mostly due to neutrons, was estimated using the MARS simulation [23-27]. The coil density was assumed to be $9 \mathrm{~g} / \mathrm{cm}^{3}$, and the thickness of the stainless steel support structure of the coil was $10 \mathrm{~mm}$. The total energy deposited at the solenoid coils including the support structure was about $0.6 \mathrm{~W}$ for a proton beam of $400 \mathrm{MeV}$ and $1 \mu \mathrm{A}$. Given a static heat load of $1 \mathrm{~W}$, the combined value is below the cryocooler total cooling power.

The curved MTS is employed to preferentially select muons in the momentum region $(\sim 20-60 \mathrm{MeV})$ low enough to be subsequently stopped in a target further downstream. In order to have a high transport efficiency for such muons, the solenoid has a large bore of $36 \mathrm{~cm}$ with an on-axis magnetic field of $2 \mathrm{~T}$. A CD magnetic field from -0.04 to $+0.04 \mathrm{~T}$ is applied in order to keep the trajectory of the low-energy muons roughly centered and to filter out other particles. The CD magnetic field can be also used to select electric charges of muons in the beam coming downstream. However, the covered arc of $36^{\circ}$ is not large enough to obtain a good separation of charges, and, therefore, contamination of oppositely charged particles

TABLE I. Design parameters of the PCS, MTS, and CD magnets. The coil thickness for the PCS is $40 \mathrm{~mm}$ upstream of $400 \mathrm{~mm}$ to increase the magnetic field. RRR is a residualresistance ratio of superconductors used.

\begin{tabular}{|c|c|c|c|}
\hline & PCS & MTS & $\mathrm{CD}$ \\
\hline \multirow[t]{2}{*}{ Conductor } & $\mathrm{Cu}$-stabilized & $\mathrm{Cu}$-stabilized & Cu-stabilized \\
\hline & $\mathrm{NbTi}$ & $\mathrm{NbTi}$ & $\mathrm{NbTi}$ \\
\hline $\begin{array}{l}\text { Conductor } \\
\text { diameter (mm) }\end{array}$ & 1.2 & 1.2 & 1.2 \\
\hline $\mathrm{Cu} / \mathrm{NbTi}$ & 4 & 4 & 4 \\
\hline $\begin{array}{l}\text { RRR } \\
\text { (R293K/R10K) }\end{array}$ & $>240$ & $>150$ & $>150$ \\
\hline Coil diameter (mm) & 900 & 480 & 460 \\
\hline Coil length (mm) & 1000 & 200 & 200 \\
\hline Coil thickness (mm) & 35 & 30 & \\
\hline Number of turns & 30000 & 4000 & 528 \\
\hline Operation current (A) & 145 & 145 & 115 (bipolar) \\
\hline Field $(\mathrm{T})$ & 3.5 & 2 & 0.04 \\
\hline Inductance $(\mathrm{H})$ & 400 & 124 & 0.04 \\
\hline Stored energy (MJ) & 5 & 1.4 & \\
\hline Quench back heater & $\begin{array}{l}1.2 \mathrm{~mm} \\
\mathrm{Cu} \text { wire }\end{array}$ & $\begin{array}{l}1.3 \mathrm{~mm} \\
\mathrm{Cu} \text { wire }\end{array}$ & \\
\hline
\end{tabular}

in the beam could not be fully eliminated, as mentioned later. The design parameters of the MTS and CD are given in Table I.

\section{MUON DETECTION}

The particles exiting the beam pipe were a mixture of electrons and positrons, pions and muons of both charges, and protons and neutrons, because of the short arc of $36^{\circ}$ coverage of the MTS. The challenge of this experiment is to measure the muon flux above such high backgrounds. Two methods were employed, the first of which measures electrons (and positrons) from muon decays and the second measures negatively charged muons. The first relies on measuring the decay time spectrum of the muon to identify and count their number by measuring a coincidence of a muon in one detector followed by an electron in a later detector. The second measures the muonic x-ray energy spectrum emitted by the negatively charged muons captured by a nucleus. The experimental setups are given in the following.

\section{A. Muon decay spectrum}

A system to measure the muon flux was made by detecting electrons (and positrons) from muon decays. The correction magnetic field $B_{\mathrm{CD}}=-0.04 \mathrm{~T}$ was set to primarily select positively charged muons. According to simulations (see Sec. V), electrons made up about $74 \%$ of the beam, with positrons the next most prevalent, making up about $10 \%$ of total population. The fractions of positive and negative muons were estimated to be about 5\% and $0.5 \%$, respectively. The fractions of neutrons and positively and negatively charged pions were estimated to be about $5 \%, 3 \%$, and $0.2 \%$, respectively.

An aluminum degrader, with a height and width both of $400 \mathrm{~mm}$, was optionally placed immediately after the beam exited the beam pipe and magnet system. Thicknesses of $0.5,1$, and $5 \mathrm{~mm}$ were used in order to select different ranges of muon momentum. From simulations, the mean momentum for muons at the end of the beam pipe and subsequently stopped were, e.g., with no degrader and a 5-mm-thick degrader, $45.2 \pm 0.2$ and $66.1 \pm 0.2 \mathrm{MeV}$, respectively. After the degrader, a copper or magnesium stopping target was sandwiched between two sets of plastic scintillator counters. The first scintillator, S1, consisted of eight channels, with a height and width of 30 and $380 \mathrm{~mm}$, respectively, and was $0.5 \mathrm{~mm}$ thick, in order to disturb the beam minimally. This counter was used to detect the passage of the initial muons. The second scintillator, S2, consisted of five channels, with a height and width of 50 and $380 \mathrm{~mm}$, respectively, and was of thickness $3.5 \mathrm{~mm}$; this was used to detect electrons from the decay of a muon. The scintillators were wrapped in reflective mylar foils and black plastic sheets to prevent light leakage. Each scintillator strip had a wavelength-shifting fiber mounted on the 


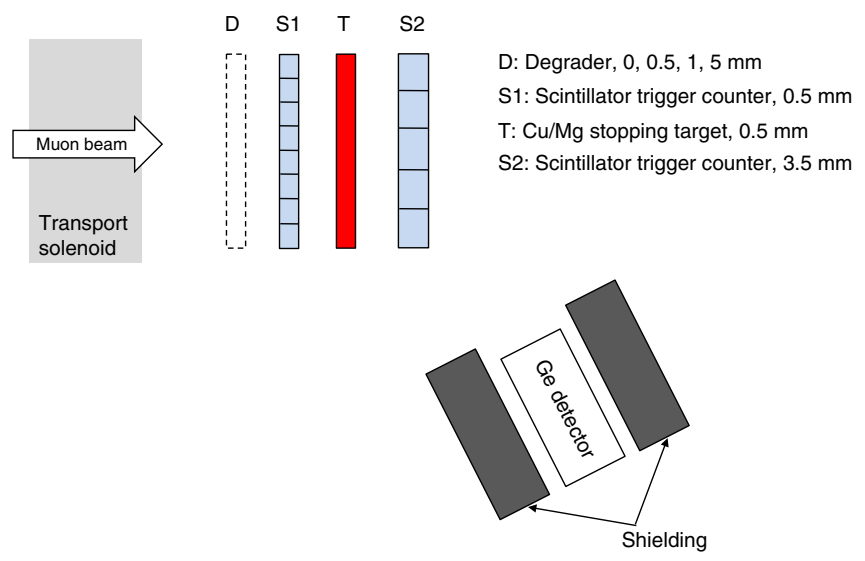

FIG. 2. Schematic of the independent detection systems for measuring the muon flux. After a $36^{\circ}$ arc of the muon beam line, the beam, including muons, exits the transport solenoid and optionally passes through an aluminum degrader $\mathrm{D}$. The beam then passes through a plastic scintillator trigger counter S1 and a target $\mathrm{T}$, where muons are stopped. $\mathrm{X}$ rays emitted by a captured muon dropping down orbital energy levels are detected in a germanium detector, which is also shielded. Electrons arising from the decay of a muon are registered in another plastic scintillator, S2.

back of it which was connected to a multipixel photon counter (MPPC) at each end. The signals from each pair of MPPCs were combined and amplified before being passed to the data acquisition (DAQ) system for processing. A schematic of the setup is shown in Fig. 2.

In order to trigger on muon decays, a "hit" recorded in S2 was required to occur 50 ns to $20 \mu$ s after the hit in S1. A hit in S1 was defined as a coincident signal in both MPPCs of a given scintillator strip where the signal value was at least eight photons, set by a discriminator. A hit in $\mathrm{S} 2$ was given by a signal of at least ten photons, higher than in $\mathrm{S} 1$ as electrons deposit more energy in the thick scintillators than slow muons in the thin scintillators. The time difference $t$ between the signals in S1 and S2 was used for further analysis in order to reconstruct the muon decay time spectrum.

\section{B. Muonic x-ray measurement}

A complementary system to measure the negative muon flux was the use of a germanium detector to measure $\mathrm{x}$ rays emitted by negatively charged muons as they drop down energy levels in a muonic atom after being captured by one of the nuclei in the target T, shown in Fig. 2. A stopping target was made of magnesium of $20 \mathrm{~mm}$ thickness, with a height and width of 80 and $370 \mathrm{~mm}$, respectively. The germanium detector is a planar type of Canberra GL0515R with a germanium crystal of an active diameter of $25.2 \mathrm{~mm}$ and depth of $15 \mathrm{~mm}$. The cryostat window, made of beryllium, was $0.15 \mathrm{~mm}$ thick. To ensure low backgrounds from particles exiting the beam line and other secondary interactions, the detector was placed off axis by $25^{\circ}$ from the beam axis and a distance of $500 \mathrm{~mm}$ from the target. The CD magnetic field of $B_{\mathrm{CD}}=+0.04 \mathrm{~T}$ was chosen to select negatively charged muons. This setup is also shown schematically in Fig. 2.

Since the detector was not far from the proton target, the neutron flux at the detector location was very high. To reduce neutrons, the germanium detector was shielded with paraffin, cadmium, and lead blocks from the outer to the inner sides. The paraffin cylindrical shielding of $100 \mathrm{~mm}$ thickness was used to decelerate fast neutrons to thermal neutrons. The cadmium shielding of $2 \mathrm{~mm}$ thickness was used to absorb thermal neutrons followed by $\gamma$-ray emission. The lead shielding of $50 \mathrm{~mm}$ thickness was used to absorb $\gamma$ rays from cadmium and other $\gamma$ rays. Furthermore, it was found that characteristic $\mathrm{x}$ rays from lead blocked the muonic $L_{\alpha} \mathrm{x}$ rays and the pionic $\mathrm{x}$ rays from magnesium. Additional shielding, which consisted of cylindrical tubes of tin, copper, and aluminum (outer to inner) were placed between the germanium detector and the lead shielding.

The signal output from the germanium detector was amplified and fed to a multichannel analyzer (MCA). The MCA was triggered by the muon stop logic signal formed by the beam hodoscopes. The energy calibration of the germanium detector was done using a ${ }^{133} \mathrm{Ba}$ source.

\section{SIMULATION}

The experimental setup was simulated in order to aid the design of the detectors, to determine efficiencies and acceptances, and to compare to the data. Two codes were used to perform the simulation: G4BEAMLINE [28] was used to simulate the hadron production and track particles through the beam line, and GEANT4 [29] was used to simulate the detectors.

G4BEAmLine was used to simulate the bulk of the experiment: the initial proton beam; the pion capture system, including the target, capture solenoid, shielding, and return yoke; and the transport system, bending magnets, and beam pipe sections. The position and momentum of all particles passing through the end of the beam line were recorded and used as input to the subsequent GEANT4 simulation.

GEANT4 is used to simulate the detector setup shown in Fig. 2. Using the output from G4BeAmLINE, the particles pass through materials constituting the various detector components, interacting with them according to formulas for energy loss, emission spectra, etc. A full optical simulation of photon production in the scintillators and detection in the MPPCs was performed. The QGSP_BERT_HP physics list in GEANT4 was used to simulate hadronic interactions, as this is designed to transport neutrons with energies as low as $20 \mathrm{MeV}$. The reconstruction and cuts described in Sec. IV as well as the signal extraction described in Sec. VI were performed on the simulated data as for the real data. 


\section{ANALYSIS METHOD}

\section{A. Analysis of muon lifetime}

The distributions of $t$ are shown in Fig. 3 for each of the five S2 channels for an example data sample (see [30] for other samples). Also shown is a fit, $N(t)$, to the data given by

$$
\begin{aligned}
N(t)= & N_{f} \exp \left(-\frac{t}{\tau_{f}}\right)+N_{s} \exp \left(-\frac{t}{\tau_{s}}\right) \\
& +N_{b 1} \sin \left(2 \pi \frac{t-\phi}{T}\right)+N_{b 2},
\end{aligned}
$$

where $t$ is the time in nanoseconds. The four components correspond to the free decay of positive muons, $f$, the decay of negative muons in the stopping target, $s$, a sinusoidal background $b 1$, and a flat background $b 2$. The muon decays rates are parametrized with a scale factor $N$ and a lifetime $\tau$. The sinusoidal background term, which comes from beam particles (mostly electrons) directly hitting the counters, has a period $T$ and a phase relative to the trigger time, $\phi$. It is due to the minor bunching of the protons in acceleration, with the rf frequency of the RCNP cyclotron. The flat background term is due to combinatorial background of beam particles which fake a signal. Known values of the lifetimes, $\tau_{f}=2196.9811 \pm 0.0022 \mathrm{~ns}$ [9] and, for a copper stopping target, the lifetime of a muonic atom of copper [31], $\tau_{s}=163.5 \pm 1.0 \mathrm{~ns}$, were fixed in the fit. The value of $T$ was also fixed to $60 \pm 5 \mathrm{~ns}$ determined by fitting the background noise in a region where no signal is expected, i.e. high $t$. The time distribution for an example data sample, with a degrader thickness of $5 \mathrm{~mm}$, is shown for each of the five S2 channels in Fig. 3. The fit to the data with the function in Eq. (7) is also shown. The fits to the data are reasonable with some distributions having values of $\chi^{2}$ per degree of freedom of about one as is the case for the first channel shown here. Channels 4 and 5 give consistently poor values, worse than channels from 1 to 3 , and so are excluded from further analysis.

The functions for the free decays of positive muons and decays of negative muons in a muonic atom of copper with parameters extracted in the fit to the data are then integrated in order to determine the number of muons in each sample. As a cross-check of the method, simulated distributions were fit with the function in Eq. (7) and the number of muons extracted. These values agreed with simply counting the number of real muons in the simulation.

The dead time of the DAQ system, due to it being busy, was calculated for each running configuration from the number of potential and good triggers. The potential triggers were those with a signal in the upstream scintillator and no corresponding signal downstream within the $50 \mathrm{~ns}$ veto window. A good trigger was defined as a potential trigger without the system being busy. The dead time varied
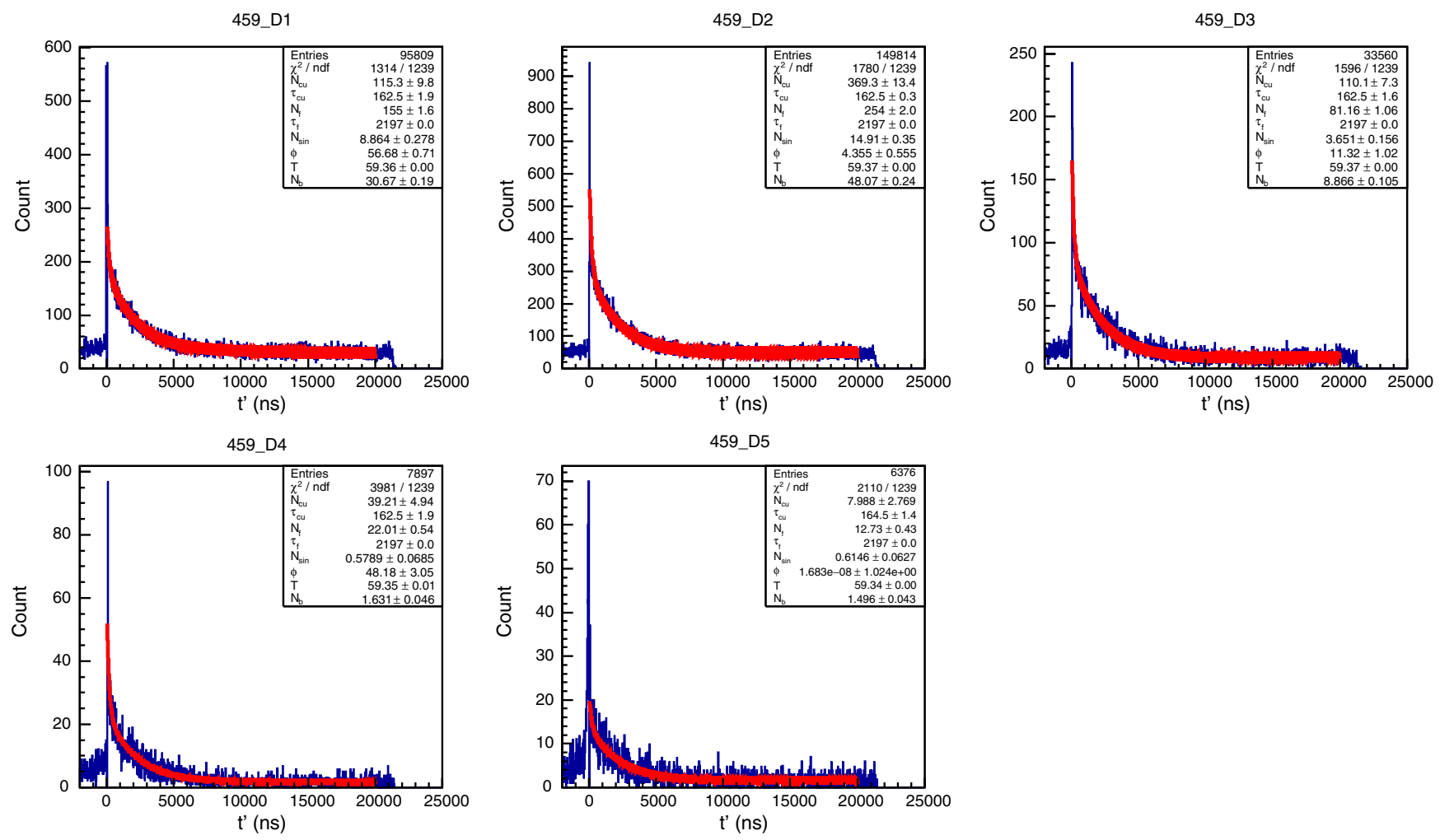

FIG. 3. Time distribution for an example data sample, with a degrader thickness of $5 \mathrm{~mm}$, for each of the five $\mathrm{S} 2$ channels. The fit to the data with the function in Eq. (7) and the parameters extracted for each distribution are also shown. 
from $38.43 \pm 0.03 \%$ when no degrader was used to $15.05 \pm$ $0.12 \%$ when a 5 -mm-thick degrader was used. The decreasing dead time with increasing degrader thickness is to be expected due to the decrease in the overall rate of beam particles entering the detector system. In order to calculate the muon flux, the data were corrected for the dead time.

In order to extract a measured value, independent of the detector setup, the muon flux was corrected for the detector acceptance and the MPPC efficiency. The detector acceptance was calculated with a simulation by determining the fraction of muons which produced the signals described previously in the two scintillator detectors. The value was $A=79.6 \pm 1.2 \%$. The efficiency of the MPPCs was determined using cosmic rays by placing the scintillator and MPPC system between a set of large scintillator paddles with the scintillation photons detected by photomultiplier tubes. By requiring a coincidence in the paddles above and below, the efficiency of an MPPC, accounting for the geometrical acceptance, was determined to be $\epsilon=0.431 \pm 0.134$. The large uncertainty comes from the lack of stability over time and the differences for different MPPCs. Given the requirement in the measurement of signals in both MPPCs for a given scintillator strip, this gives an efficiency of $\epsilon^{2}=18.6 \pm 11.6 \%$. The efficiency of the MPPC system is the dominant systematic uncertainty.

Other uncertainties considered were related to the fitting procedure and extraction of the integrated number of the electrons and positrons from muon decays, $N_{e}$, in their time spectra. The lower bound on the fit and the binning of the data were varied. The uncertainty on the number of free positive muon decays was about $1.1 \%$, where, for negative muons decaying in copper, it was about $56 \%$. The large uncertainty for the results of decays in copper arises due to the sensitivity of the fit at small times where the decays in copper are concentrated.

The total number of muons stopped in the target, $N_{\mu}$, measured by the decay electrons and positrons is given by

$$
N_{\mu}=\frac{N_{e}}{A \cdot \epsilon^{2}},
$$

where $A$ and $\epsilon$ are the detector acceptance and the MPPC efficiency, respectively. The data samples with the same degrader thickness give consistent results, demonstrating the control of the analysis with time. The muon rate also decreases with increasing degrader thickness, consistent with the expectation. These two qualitative conclusions were observed for both free decays of positive muons and negative muons decaying in a muonic atom of copper.

\section{B. Analysis of muonic x-ray measurement}

The muonic $\mathrm{x}$-ray measurements on magnesium were done for three data sets [32]. The list of the data sets is shown in Table II, where different proton beam currents
TABLE II. Data sets for muonic x-ray measurements with different proton beam currents.

\begin{tabular}{lcccc}
\hline \hline $\begin{array}{l}\text { Data } \\
\text { name }\end{array}$ & $\begin{array}{c}\text { Measurement } \\
\text { time }(\mathrm{s})\end{array}$ & $\begin{array}{c}\text { Proton beam } \\
\text { current }(\mathrm{pA}) \\
\left(I_{p}\right)\end{array}$ & $\begin{array}{c}\text { Muonic } K_{\alpha} \\
\mathrm{x}-\text { ray events } \\
\left(N_{2 \rightarrow 1}\right)\end{array}$ & $\begin{array}{c}\text { Muonic } L_{\alpha} \\
\mathrm{x} \text {-ray events } \\
\left(N_{3 \rightarrow 2}\right)\end{array}$ \\
\hline Data1 & $1.0 \times 10^{4}$ & 59 & $23 \pm 6$ & $46 \pm 13$ \\
Data2 & $1.1 \times 10^{4}$ & 134 & $72 \pm 12$ & $141 \pm 22$ \\
Data3 & $1.0 \times 10^{4}$ & 435 & $212 \pm 21$ & $318 \pm 48$ \\
\hline \hline
\end{tabular}

were used. The x-ray spectra for each data set are shown in Fig. 4, where muonic $K_{\alpha}$ and $L_{\alpha}$ x rays were measured together with pionic $\mathrm{x}$ rays. The energy calibration was done using a ${ }^{133} \mathrm{Ba}$ source.

The muonic x-ray peaks were fit with a Gaussian and a constant background to obtain the total number of events of muonic $\mathrm{x}$ rays. The energy dependence of the x-ray peak width was found from the measured data to be

$$
\sigma=0.0196 \sqrt{E(\mathrm{keV})+57} .
$$

The numbers of muonic x-ray events are summarized in Table II.

The total number of negatively charged muons stopped in the target, $N_{\mu^{-}}$, was determined using the following:

$$
N_{\mu^{-}}=\frac{N_{i \rightarrow j}}{P_{i \rightarrow j} \times(\Omega / 4 \pi) \times \varepsilon},
$$

where $N_{i \rightarrow j}$ and $P_{i \rightarrow j}$ are the number and the emission probability, respectively, of the muonic $\mathrm{x}$ ray in transition from the muonic atomic state of $i$ to that of $j ; P_{i \rightarrow j}$ is known [33] and listed in Table III. The quantity $(\Omega / 4 \pi)$ is the solid angle of the germanium detector, and $\varepsilon$ is the efficiency of the germanium detector. Their combination $\Omega_{D}=(\Omega / 4 \pi) \times \varepsilon$ can be estimated from GEANT4 simulations taking into account the energy dependence of absorption by material and that of detection in germanium. The values $\Omega_{D}=(1.28 \pm 0.05) \times 10^{-5}$ for $K_{\alpha}$ x rays and $\Omega_{D}=(2.77 \pm 0.12) \times 10^{-5}$ for $L_{\alpha} \times$ rays were obtained.

The simulation was validated by the measurements with the standard calibration source of ${ }^{133} \mathrm{Ba}$ with its known absolute strength. The source was placed $200 \mathrm{~mm}$ away from the germanium detector. The comparison between the simulation and the measurement gave uncertainties of $2 \%$ and $6 \%$ for the reconstruction of the $K_{\alpha}$ and $L_{\alpha}$ x rays, respectively.

\section{RESULTS AND MEASUREMENT OF MUON BEAM INTENSITY}

\section{A. Results from muon lifetime analysis}

The final number needed in order to extract the total muon flux is the number of muons which pass through the 


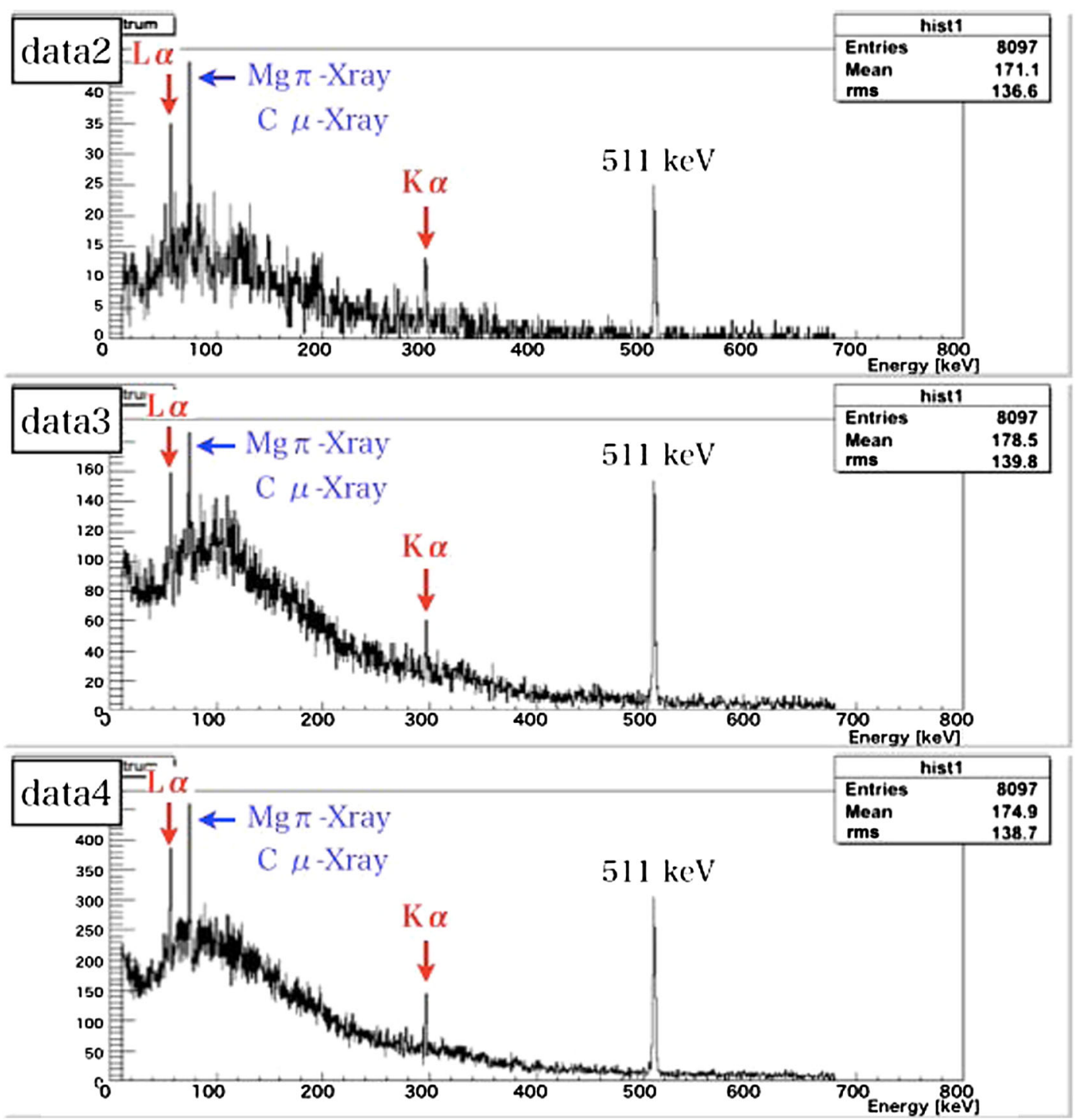

FIG. 4. Muonic x-ray spectra for different data sets.

degrader, subsequently stop, and are measured in the detector system. The simulation started from the pion production was used to get the muon stopping distribution in the stopping target. Figure 5 shows the momentum distribution of muons stopped in the target from the simulation. From the simulation, the probability of the muons stopping was determined to be at most $F_{s}=8 \%$, depending on the degrader thickness, and includes both positive and negative muons.

TABLE III. Energy and emission probability for different muonic $\mathrm{x}$ rays on magnesium.

\begin{tabular}{lcc}
\hline \hline $\begin{array}{l}\text { Muonic x ray } \\
(i \rightarrow j)\end{array}$ & Energy & Probability \\
\hline$E_{i \rightarrow j}(\mathrm{keV})[34]$ & $P_{i \rightarrow j}(\%)[33]$ \\
\hline$L_{\alpha}(2 \rightarrow 1)$ & 296.4 & $(79.6 \pm 0.7)$ \\
\hline \hline
\end{tabular}

Given an expected beam design current of $1 \mu \mathrm{A}$, the final muon flux in muons per second is given by

$$
J_{\mu}=\frac{N_{\mu}}{I_{p} \cdot F_{s} \cdot D \cdot L}
$$

where $I_{p}$ is the proton beam current in $\mu \mathrm{A}, D$ is the duration of the run, and $L$ is the live time, i.e. the dead time subtracted from $100 \%$.

The data samples taken with different degrader thicknesses are consistent with each other, and so averaging over these values gives a final flux of $J_{\mu}=(4.2 \pm 1.1) \times 10^{8}$ muons s $^{-1}$, which corresponds to an efficiency for full beam power $(400 \mathrm{~W})$ of $J_{\mu} / W_{p}=(10.4 \pm 2.7) \times 10^{5}$ muons s$^{-1} \mathrm{~W}^{-1}$. This value is far in excess of any other muon source, i.e. is by far the most efficient muon beam produced. 


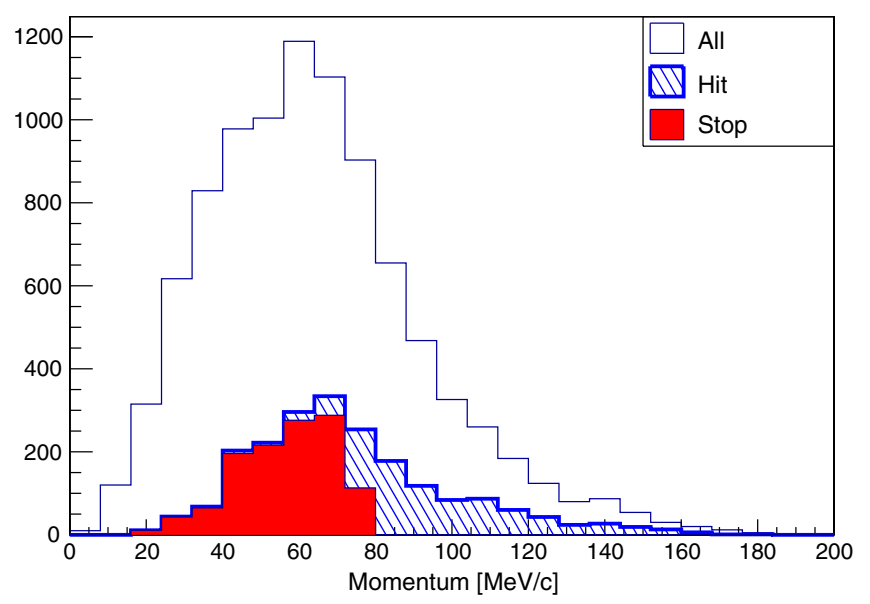

FIG. 5. Momentum distributions for all muons in a beam (white), the muons hitting the trigger counters (blue), and those stopped in the target (red).

\section{B. Results from x-ray measurements}

From the three data sets with different proton beam currents, the muon beam intensity of negative muons, $J_{\mu^{-}}$, was obtained to be

$$
J_{\mu^{-}}=\frac{N_{\mu^{-}}}{I_{p} \cdot F_{s} \cdot D \cdot L}
$$

where $I_{p}$ is the proton beam current in $\mu \mathrm{A}, D$ is the duration of the run, and $L$ is the live time. $N_{\mu^{-}}$is the total number of muons stopped in the target, given in Eq. (10). The measurements of $K_{\alpha}$ and $L_{\alpha}$ x rays were combined. For a maximum proton beam current of $1 \mu \mathrm{A}$ at RCNP, a muon yield of $J_{\mu^{-}}=(3.6 \pm 0.4) \times 10^{7}$ muons s ${ }^{-1}$, which is the world's highest, was obtained. The muon yield per beam power is $J / W_{p}=(9.0 \pm 1.0) \times 10^{4}$ muons s $\mathrm{W}^{-1}$, an improvement of about 1000 over existing facilities. GEANT simulations with QGSP_BERT give about $J_{\mu^{-}}=6.5 \times 10^{7}$ muons s$^{-1}$, which is larger than the measured yield. This could be due to the hadron code in GEANT simulations.

\section{SUMMARY}

The world's most efficient muon beam has been produced at the MuSIC facility in Osaka, Japan. The muon yield per beam power was measured to be (for $\mu^{+}$and $\mu^{-}$) $(10.4 \pm 2.7) \times 10^{5}$ muons s ${ }^{-1} \mathrm{~W}^{-1}$ and (for $\mu^{-}$only) $(9.0 \pm 1.0) \times 10^{4}$ muons s${ }^{-1} \mathrm{~W}^{-1}$, over a factor of 1000 higher than current facilities. Given a maximum beam power of $400 \mathrm{~W}$, rates of about $4 \times 10^{8}$ muons s$^{-1}$ are achievable.

The final configuration of the MuSIC facility is planned to have the MTS cover an arc of $180^{\circ}$ instead of $36^{\circ}$. In this configuration, better discrimination of wrong-signed charged particles of $<10^{-4}$ would be expected with almost no loss of muons of interest.
Currently, before realizing the final configuration, an extension of the muon beam line composing of normalconducting dipole and quadrupole magnets with a dc separator was constructed. It is now being commissioned to provide a continuous muon beam for applications in material science. The details of the performance of this beam line will be reported in a separate publication.

The increase in efficiency reported here arises principally due to the use of a novel superconducting solenoid magnet system to capture pions produced at a target. This demonstration in increased efficiency will be utilized in future muon experiments in order to maximize the flux of muons and hence search for charged lepton flavor violation.

\section{ACKNOWLEDGMENTS}

This work is supported in part by the Japan Society of the Promotion of Science (JSPS) KAKENHI Grant No. 25000004. The support of the Science and Technology Facilities Council, United Kingdom is acknowledged. M.W. acknowledges the support of DESY and the Alexander von Humboldt Stiftung.

[1] Y. Kuno and Y. Okada, Muon decay and physics beyond the standard model, Rev. Mod. Phys. 73, 151 (2001).

[2] R. H. Bernstein and P. S. Cooper, Charged lepton flavor violation: An experimenter's guide, Phys. Rep. 532, 27 (2013).

[3] J. Adam et al. (MEG Collaboration), New Constraint on the Existence of the $\mu^{+} \rightarrow e^{+} \gamma$ Decay, Phys. Rev. Lett. 110, 201801 (2013).

[4] Y. Kuno (on behalf of the COMET Collaboration), A search for muon-to-electron conversion at J-PARC: the COMET experiment, Prog. Theor. Exp. Phys. (2013) $022 \mathrm{C} 01$.

[5] R. J. Abrams et al. (Mu2e Collaboration), Mu2e conceptual design report, arXiv:1211.7019.

[6] A. Blondel et al., Research proposal for an experiment to search for the decay $\mu \rightarrow$ eee, arXiv:1301.6113.

[7] D. M. Webber et al. (MuLan Collaboration), Measurement of the Positive Muon Lifetime and Determination of the Fermi Constant to Part-per-Million Precision, Phys. Rev. Lett. 106, 041803 (2011).

[8] G. W. Bennett et al. (Muon $g-2$ Collaboration), Final report of the E821 muon anomalous magnetic moment measurement at BNL, Phys. Rev. D 73, 072003 (2006).

[9] J. Beringer et al. (Particle Data Group), Review of particle physics, Phys. Rev. D 86, 010001 (2012), and 2013 partial update for the 2014 edition.

[10] R. M. Carey et al. (New Muon ( $g-2)$ Collaboration), The new $(g-2)$ experiment: a proposal to measure the muon anomalous magnetic moment to $\pm 0.14 \mathrm{ppm}$ precision, see http://lss.fnal.gov/archive/test-proposal/0000/fermilabproposal-0989.pdf. 
[11] N. Saito (J-PARC $g-2 / E D M$ Collaborations), A novel precision measurement of muon $g-2$ and EDM at JPARC, AIP Conf. Proc. 1467, 45 (2012).

[12] Muon Collider and Neutrino Factory Overview, edited by M. A. Palmer, ICFA Beam Dynamics Newsletter Vol. 55 (2011), http://icfa-usa.jlab.org/archive/newsletter/ icfa_bd_nl_55.pdf.

[13] S. J. Blundel, Spin-polarized muons in condensed matter physics, Contemp. Phys. 40, 175 (1999).

[14] A. Yaouanc and P. Dalmas de Réotier, Muon Spin Rotation, Relaxation and Resonance: Applications to Condensed Matter (Oxford University Press, New York, 2011).

[15] H. Daniel, Application of $X$ rays from negative muons, Nucl. Instrum. Methods Phys. Res., Sect. B 3, 65 (1984).

[16] M. K. Kubo, H. Moriyama, Y. Tsuruoka, S. Sakamoto, E. Koseto, T. Saito, and K. Nishiyama, Non-destructive elemental depth-profiling with muonic X-rays, J. Radioanal. Nucl. Chem. 278, 777 (2008).

[17] M. Yoshida, M. Fukuda, K. Hatanaka, Y. Kuno, T. Ogitsu, A. Sato, and A. Yamamoto, Superconducting solenoid magnets for the MuSIC project, IEEE Trans. Appl. Supercond. 21, 1752 (2011).

[18] R. M. Dzhilkibaev and V. M. Lobashev, Yad. Fiz. 49, 622 (1989) [On the search for $\mu \rightarrow e$ conversion on nuclei. (In Russian), Sov. J. Nucl. Phys. 49, 384 (1989)].

[19] R. Djilkibaev and V. M. Lobashev, The solenoid muon capture system for the MELC experiment, AIP Conf. Proc. 372, 53 (1996).

[20] S. Geer, Neutrino beams from muon storage rings: Characteristics and physics potential, Phys. Rev. D 57, 6989 (1998).

[21] T. Prokscha, E. Morenzoni, K. Deiters, F. Foroughi, D. George, R. Kobler, A. Suter, and V. Vrankovic, The new $\mu E 4$ beam at PSI: A hybrid-type large acceptance channel for the generation of a high intensity surface-muon beam, Nucl. Instrum. Methods Phys. Res., Sect. A 595, 317 (2008).

[22] I. Miura, The research center for nuclear physics ring cyclotron, in Proceedings of the 15th Particle Accelerator
Conference, PAC-1993, Washington, DC, 1993 (IEEE, New York, 1993), p. 1650, http://accelconf.web.cern.ch/ AccelConf/p93/PDF/PAC1993_1650.PDF.

[23] N. V. Mokhov, Report No. Fermilab-FN-628, 1995, http://lss.fnal.gov/archive/test-fn/0000/fermilab-fn-0628 .pdf.

[24] N. V. Mokhov and O. E. Krivosheev, Report No. FermilabConf-00/181, 2000, http://lss.fnal.gov/archive/2000/conf/ Conf-00-181.pdf.

[25] N. V. Mokhov, Report No. Fermilab-Conf-03/053, 2003, http://lss.fnal.gov/archive/2003/conf/Conf-03-053.pdf.

[26] N. V. Mokhov et al., Report No. Fermilab-Conf-04/053, 2004, http://lss.fnal.gov/archive/2004/conf/fermilab-conf04-053-ad.pdf.

[27] https://mars.fnal.gov.

[28] T. J. Roberts and D. M. Kaplan, G4beamline simulation program for matter-dominated beamlines, in Proceedings of the 22nd Particle Accelerator Conference, PAC-2007, Albuquerque, NM (IEEE, New York, 2007), p. 3468, http:// ieeexplore.ieee.org/document/4440461/.

[29] S. Agostinelli et al., Geant4-a simulation toolkit, Nucl. Instrum. Methods Phys. Res., Sect. A 506, 250 (2003); J. Allison et al., Geant4 developments and applications, IEEE Trans. Nucl. Sci. 53, 270 (2006).

[30] S. L. Cook, Ph.D. thesis, University College London, 2014, available at http://www.hep.ucl.ac.uk/theses/SamCook .pdf.

[31] T. Suzuki, D. F. Measday, and J. P. Roalsvig, Total nuclear capture rates for negative muons, Phys. Rev. C 35, 2212 (1987).

[32] Y. Hino, Master's thesis (in Japanese), Osaka University, 2012, available at http://www-kuno.phys.sci.osaka-u.ac.jp/ papers/m-thesis/fy2012_hino_m-thesis.pdf.

[33] P. Vogel, Muonic cascade: General discussion and application to the third-row elements, Phys. Rev. A 22, 1600 (1980).

[34] A. Suzuki, Relative Intensities of $K$ and $L$ Series of Muonic X Rays, Phys. Rev. Lett. 19, 1005 (1967). 